\title{
Missed Injuries in Trauma Patients; The Value of a Diagnostic Thoracotomy or Thoracoscopy During Surgical Stabilisation of Rib Fractures.
}

Tess Wemeijer ( $\square$ tesswemeijer@hotmail.com )

MST: Medisch Spectrum Twente

Wim Hogeboom

MST: Medisch Spectrum Twente

Pascal Steenvoorde

MST: Medisch Spectrum Twente

Dominique S. Withaar

MST: Medisch Spectrum Twente

Reinier de Groot

MST: Medisch Spectrum Twente

\section{Research Article}

Keywords: Surgical Stabilization of Rib Fractures, missed diagnosis, blunt trauma, diaphragm rupture

Posted Date: June 14th, 2021

DOI: https://doi.org/10.21203/rs.3.rs-268188/v1

License: (c) (i) This work is licensed under a Creative Commons Attribution 4.0 International License. Read Full License 


\section{Abstract}

\section{Purpose}

Over the last decade Surgical Stabilisation of Rib Fractures (SSFR) gained popularity in our hospital. With increased numbers, we noted that frequently injuries were missed during primary/secondary survey and radiological imaging, that were found during the surgical procedure. With this observation, the research question was formulated: What is the value of diagnostics thoracotomy or thoracoscopy during surgical stabilisation of rib fractures?

\section{Methods}

In a single centre, retrospective study between February 2010 till December 2019, trauma patients who underwent Surgical Stabilization of Rib Fractures (SSFR) and an inspection thoracotomy were included. All radiological injuries were compared with intraoperative findings. Missed injuries that were discovered during the surgical procedure that weren't analysed during primary/secondary survey or on radiological imaging were recorded and retrospectively analysed by an independent radiologist.

\section{$\underline{\text { Results }}$}

51 patients were included. Eight patients had additional injuries; all had a diaphragmatic rupture, one patient had an attending stomach laceration, another patient had an attending significant lung laceration in need of surgical repair. Only 13 out of 56 diaphragm rupture CT-signs were confirmed, therefore still $77 \%$ of signs could not be confirmed by initial radiological findings.

\section{$\underline{\text { Conclusion }}$}

With the recent shift towards surgical stabilisation of rib fractures, an inspection thoracoscopy or thoracotomy during SSFR should be considered to minimize the incidence of missed intrathoracic injuries requiring early or late surgical treatment.

\section{Background}

In the last two decades, there has been a change in management of rib fractures. Benefits of surgical stabilization over internal pneumatic stabilisation in traumatic flail chest patients has been proven. ${ }^{1-3)}$ Worldwide, Surgical Stabilisation of Rib Fractures (SSRF) has gained popularity and nowadays it is frequently performed in patients with severe chest wall injuries. ${ }^{4)}$ SSFR indication has recently been generalised to trauma patients with three or more rib fractures without flail, with optimistic results in pain scores and self-reported respiratory disability. ${ }^{5}$ ) With this shift towards surgical management, additional injuries are being diagnosed during intraoperative inspection that weren't detected with primary radiological imaging. Missed injuries in trauma patients are a well-known obstacle in acute care. Incidence rates for missed injuries and delayed diagnosis range from $1.3-39 \%$ in patients suffering blunt 
trauma. ${ }^{6)}$ For example, despite an overall incidence of diaphragm injury of 0.46 percent in trauma patients ${ }^{7}$ ), it has been found in $3-8 \%$ of patients undergoing surgical exploration. ${ }^{8)}$ We hypothesize that with the shift towards SSRF, an inspection through thoracoscopy or thoracotomy should be considered to diagnose otherwise missed intrathoracic injuries needing surgical intervention.

\section{Methods}

A single centre, retrospective study was performed in a Dutch Level one Trauma Centre. All cases who underwent an exploring thoracotomy during SSRF from February 2010 till December 2019 were included. No exclusions criteria were applied.

Due to the retrospective design and the anonymous processing of information and results, approval by the medical ethics committee was waived.

All radiological injuries were compared with intraoperative findings. Missed injuries that were discovered during the surgical procedure that weren't analysed during primary/secondary survey or by radiological imaging were recorded. Additionally age, sex and ISS were stated. The Injury Severity Score (ISS) at admission was formulated using the global method, listing the three most severe injuries in different body regions on a 6 point scale $(1=$ minor injury, $6=$ maximal injury $) .{ }^{9)}$ Also trauma mechanism, number of rib fractures and other primary injuries were listed. Nonparametric statistical tests were used to estimated significance.

In retrospect, CT-scans from trauma patients with missed injuries were analysed again by an independent radiologist to compare perioperative findings with the primary radiological images. Due to the lengthy time period (2010-2020) the analysed CT-scans were performed on different models of CT scanners. The oldest scans were performed on a 64-slice scanner (either Toshiba Aquilion 64 or Siemens Somatom Definition AS), as the more recent scans were performed on a 256-slice Dual-Source scanner (Siemens Somatom Definition Flash). The Toshiba Aquilion 64 was installed on June 2007 and replaced with a second Siemens Somatom Defenition Flash on January 2016. The first Siemens Somatom Defination Flash was installed on the Emergency ward on October 2014. Because this study was preformed in a level one Trauma Center, some patients were referred from other hospitals with CT-scans made on a Siemens Somation Defination AS with an unknown installation date.

All patients underwent a contrast-enhanced CT of the thorax (and abdomen) (either in the arterial or portal-venous phase, depending on the chosen scan protocol at the time of trauma) with full visualisation of the complete diaphragmatic dome. All available reconstructions were analysed (axial, coronal and sagittal reformatted images with 2 or $3 \mathrm{~mm}$ slice thickness) together with all available settings (soft tissue setting, lung setting, bone setting).

Among other things, all scans were analysed for the various signs of diaphragmatic rupture described by Panda et al in 2014, see Table 1. ${ }^{10)}$ Such as, a Collar sign (the visualization of a focal, waist-like constriction of the herniating abdominal viscus at the level of the torn diaphragm), Dependent-viscera 
sign (when the upper one-third of the liver abutted the posterior ribs on the right side or if the stomach or bowel abutted the posterior ribs or lay posterior to the spleen on the left side) and Dangling diaphragm sign (present if the free edge of the torn diaphragm was visible as it curled inwards towards the center of the abdomen, away from or at near right angles to the chest wall). The perioperative found diaphragm ruptures were classified following the description by Williams et al, see Table $2 .{ }^{11)}$

\section{Results}

In total 51 patients who underwent SSRF and an exploration thoracotomy or thoracoscopy were included. Indication for surgical treatment of the rib fractures differed between flail chest, haemothorax, pneumothorax, unable to detached from the ventilator and deformation of the thorax. Eleven patients of the total cohort had diaphragm injuries. Three patients with diaphragm injuries were diagnosed with primary radiological examination. Eight patients of the total of eleven patients had diaphragmatic injuries that were discovered by inspection during surgery. These eight diaphragmatic injuries weren't analysed during primary/secondary survey or at radiological imaging prior to the surgical treatment $(16.7 \%)$. The average age of all the included patients was 59 years old and 13 patients were female. The mean primary ISS at admission was 27 (range 9-57) in the complete cohort with no difference between patients with or without missed diaphragmatic injuries. The characteristics of the patients are listed below (Table 3).

Table 1

The CT-signs for diaphragmatic ruptures.

\section{The various signs of diaphragmatic rupture described by Panda et al:}

- Discontinuous diaphragm sign

- Collar sign

- Dependent-viscera sign

- Dangling diaphragm sign

- Organ herniation or hump sign due to a partial liver herniation

- Thickening of the diaphragm

- Continuous injury on either side of the diaphragm 
Table 2

Characteristics of diaphragm ruptures.

Diaphragm ruptures classified by Williams et al:

- Grade 1: Contusion

- Grade 2: Laceration $<2 \mathrm{~cm}$

- Grade 3: Laceration 2-10cm

- Grade 4: Laceration $>10 \mathrm{~cm}$ and tissue loss of $<25 \mathrm{~cm}^{2}$

- Grade 5: Laceration and tissue loss of $>25 \mathrm{~cm}^{2}$

Table 3

Characteristics of cohort. * Average age in years and full range of ages. ** Average in rib fractures and full range of fractures. *** Exact number with (percentages). **** Intra-abdominal injury (requiring laparotomy).

\begin{tabular}{|c|c|c|c|}
\hline Parameters & $\begin{array}{l}\text { Without additional injuries found } \\
\text { during surgery = } 43\end{array}$ & $\begin{array}{l}\text { Additional injuries found } \\
\text { during surgery }=8\end{array}$ & $\begin{array}{l}\text { Total } \\
=51\end{array}$ \\
\hline Men/Women & $32 / 11$ & $6 / 2$ & $38 / 13$ \\
\hline Age* & $61 y(39-83)$ & $53 y(22-78)$ & $59.4 y$ \\
\hline ISS & 27.3 (Range 9-57) & \multicolumn{2}{|l|}{26.75 (Range 9-50) } \\
\hline $\begin{array}{l}\text { Average number of } \\
\text { rib fractures }{ }^{\star \star}\end{array}$ & $10(2-24)$ & $9(3-13)$ & 9.6 \\
\hline Trauma mechanism & $3(7)$ & $0(-)$ & $3(6)$ \\
\hline \multirow{3}{*}{$\begin{array}{l}\text { Pedestrian vs car } \\
\text { Cyclist vs car }\end{array}$} & $10(24)$ & $0(-)$ & \multirow{2}{*}{$\begin{array}{l}10 \\
(20)\end{array}$} \\
\hline & $8(19)$ & $4(50)$ & \\
\hline & $2(5)$ & $0(-)$ & $\begin{array}{l}12 \\
(24)\end{array}$ \\
\hline \multirow{2}{*}{$\begin{array}{l}\text { Motorbike accident } \\
\text { Fall from height }\end{array}$} & $16(38)$ & 3 (33) & $2(4)$ \\
\hline & $4(10)$ & $1(11)$ & $\begin{array}{l}19 \\
(37)\end{array}$ \\
\hline Otherwise. & & & $5(10)$ \\
\hline Primary injuries & 6 & 4 & 10 \\
\hline Pneumothorax & 10 & 3 & 13 \\
\hline Haemothorax & $11(1)$ & $4(0)$ & 15 \\
\hline $\begin{array}{l}\text { Intra-abdominal } \\
\text { injury } \star \star \star \star\end{array}$ & 13 & 3 & 16 \\
\hline Neurocranial injury & & & \\
\hline
\end{tabular}


All eight patients with a missed injurie had a diaphragmatic rupture differ from grade 2 to grade 4 . One patient had an attending stomach laceration, another patient had an attending lung laceration due to a rib perforation without pneumothorax. Three out of eight diaphragmatic ruptures were found left-sided, five were right-sided. All missed diaphragmatic injuries were easily diagnosed during thoracic inspection through thoracotomy on the side that the SSFR took place, only one patient of the complete cohort underwent an inspection thoracoscopy with non-additional injuries due to the availability of a thoracic surgeon at the time of the SSRF procedure. Therefore 50 patients underwent a lateral thoracotomy instead of thoracoscopy. Surgical Stabilization of Rib Fractures was performed using the following techniques: straight plates with screws, intramedullary splints, Vicryl sutures or a combination depending on the characteristics of the fractures. Additional diaphragm injuries were treated with sutures, the use of a surgical mesh or a combination of both.

During retrospective inspection by an independent radiologist only 13 out of a total of 56 possible CTsigns for diaphragm rupture in the eight patients with additional injuries, were seen on the CT-scans made during primary assessment (Table 4). From the eight patients with additional found diaphragm injuries two were scanned on the Toshiba Acquilon 64 CT-scanner, four on the Siemens Somatom Definition Flash and another two were referred from another hospital with a CT-scan made on a Siemens Somation Definition. Due to the small numbers, no statistic analysis could be performed and we concluded that there was no difference in sensitivity between the different CT-scanners and the scans made during the early inclusions and recent inclusions. One patient with a grade 2 rupture of the diaphragm on the right side had no positive CT-signs at all. Even after sharing the perioperative findings with the radiologist, still $77 \%$ of the signs could not be confirmed by radiological findings. All injuries that were described during primary radiological imaging were confirmed during surgery, so no false positive diagnoses were found in this retrospective study. 
Table 4

Results of diaphragmatic rupture signs at CT-scan: *I. Discontinuous sign, II. Collar sign, III. Dependentviscera sign, IV. Dangling diaphragm sign, V. Organ herniation or liver hump, VI. Thickening, VII.

Continuous injury on either side of the diaphragm. $+=$ Retrospectively confirmed, $-=$ Retrospectively absent.

\begin{tabular}{|c|c|c|c|c|c|c|c|c|}
\hline \# & Injury & ן & II & III & IV & V & VI & VII \\
\hline 1 & Diaphragm rupture grade 3 left side & - & - & - & - & - & + & + \\
\hline 2 & Diaphragm rupture grade 3 right side & - & - & - & - & + & - & + \\
\hline 3 & Diaphragm rupture grade 4 left side & + & - & - & - & - & - & + \\
\hline 4 & Diaphragm rupture grade 2 right side & - & - & - & - & - & - & - \\
\hline 5 & Diaphragm rupture grade 4 right side & - & - & + & - & + & - & - \\
\hline 6 & Diaphragm rupture grade 2 right side & - & + & + & - & + & - & - \\
\hline 7 & $\begin{array}{l}\text { Diaphragm rupture grade } 2 \text { left side and stomach laceration } \\
\text { of } 1 \mathrm{~cm}\end{array}$ & - & - & - & - & - & + & - \\
\hline 8 & $\begin{array}{l}\text { Diaphragm rupture grade } 2 \text { right side and lung perforation < } \\
1 \mathrm{~cm}\end{array}$ & - & - & - & - & - & - & + \\
\hline
\end{tabular}

Mortality rates in these trauma patients turned out to be high, six of the total 51 patients (11.8\%) didn't survive the first month after their accident. Of the deceased patients, one had additional injuries that wasn't discovered during primary CT-scans. It concerned patient number 4, he was involved in a car accident and had a radiologically missed diaphragmatic rupture grade 4 on the left side. Due to the severe thorax deformation, extreme obesity and an infected seroma in the thorax he could not be disconnected from respiratory support after surgery and passed away due to sepsis.

Eighteen of the total cohort had intra-abdominal injuries, with a mean primary ISS of 36 (range 18-57). Only one patient was in need of acute exploration and treatment by laparotomy. The remaining seventeen were treated conservatively. Sixteen patients had some sort of neurocranial injury, with a mean primary ISS of 35 (range 17-57). Thirteen patients had a haemothorax, causing a mean ISS 26 (range 9-50), with an indication for surgical treatment. Five of them were identified as caused by a minor arterial injury and one by a major vene injury due to dissection of the Azygos vene. Three of the haemothorax patients were caused by a long laceration. In four cases no clear cause was found.

\section{Discussion}

In our retrospective study, eight of 51 patients (16.7\%) had undiagnosed diaphragm / chest / abdominal injuries during primary, secondary and radiological examinations. This is conform previous described incidence rates. All initially missed injuries were easily detected during thoracic inspection by thoracotomy or thoracoscopy and were surgically treated during the SSFR procedure. All the initially missed injuries were categorised as major or life threatening injuries according to Pfeiffer et al see 
Table 5, so early discovery contributes to enhancement of treatment. ${ }^{6)}$ Retrospectively only 13 out of 56 CT-signs are positive for diaphragmatic ruptures, so even with the perioperative knowledge, still $77 \%$ of signs could not be confirmed by radiological findings. One patients didn't have any positive CT-signs at all, with the given information, one may conclude that a diaphragm rupture cannot be excluded by a negative scan in trauma patients with rib fractures. One of the possible explanations could be found in the small difference in Hounsfield Units (HU) between the diaphragm and the adjacent visceral tissue (either the liver, spleen of stomach directly beneath the diaphragm or the (frequently seen) volume loss of the lung) on the side of the injury. In this respect, there was no clear difference between arterial and portal-venous scans. Combining this with a variable thickness of the diaphragm in both injured and healthy patients, proved to have a negative effect on visualisation of several of the described signs of diaphragm injury, especially the discontinuous diaphragm sign (without herniation) and the thickening of the diaphragm. Therefore we recommend, based on our experience, to perform a thoracic inspection during SSFR in trauma patients to prevent secondary surgical treatments and chronic complications due to missed injuries, until CT-scans and techniques significantly improve to diagnose diaphragm injuries.

\section{Table 5}

Missed injuries selected at level of severity.

\section{Missed injuries based on level of severity described by Pfeifer et al:}

- Minor injuries: Hand, wrist, foot, ankle, forearm, uncomplex soft tissue injuries and fractures, rupture of ligaments and muscle tendons.

- Major injuries: Skull injuries, neurological and arterial lesions, liver, spleen, and intestinal lacerations, femoral, humeral, pelvic, and spine fractures and dislocations.

- Life threatening injuries: injuries of main vessels in thorax, haemothorax and pneumothorax.

A missed diaphragmatic rupture tends to become larger, and herniation of abdominal organs becomes more likely, particularly if the rupture is left-sided. Small right-sided injuries may remain stable due to the liver that tamponades the defect, preventing colon herniation. Nevertheless, repair of all left-sided and most right-sided diaphragmatic injuries should be performed when recognized due to the tendency to increase. ${ }^{11)}$ Therefore, surgical treatment becomes difficult and more complications occur, when the rupture becomes chronic. A mortality rate of $30-60 \%$ has been described in patients with intrathoracic visceral herniation and strangulation after a missed diaphragmatic injury. ${ }^{10)}$ With that, early detection of intrathoracic injuries contributes to increased survival rates and quality of life in trauma patients. Multiple studies and consensus statements have mentioned the possible benefits of adding a intrathoracic inspection during SSFR. ${ }^{12}$ ) Early evacuation of residual haemothorax during thoracic inspection by thoracoscopic surgery has proven to decrease the risk of infections, discomfort and shorten length of hospital stay among other advantages such as the possibility to directly repair lung lacerations and other intra-thoracic injuries. ${ }^{13,14)}$ Other studies suggest that the with the use of thorascopic inspection, better visualisation of the fracture locations help to determine the optimal incision placement and therefore enhance minimally invasive methods with smaller incisions sides and less muscle loss. ${ }^{12,13)}$ A complete thorascopic approach has only been performed in study design and is not suitable outside the context of 
research due to limitations of adequate tools. But despite these potential benefits more data has to be collected to support and recommend the routine use of thoracoscopy (or thoracotomy) during SSFR. ${ }^{12}$ )

This study concerns trauma patients with severe injuries, causing a mean ISS of 28 points. Because of the major injuries in this cohort, intrathoracic injuries are more likely to occur. Therefore, the incidence of missed injuries, such as diaphragm ruptures, are higher and the diagnostic value of an inspection thoracoscopy or thoracotomy increases. With the recent shift towards surgical management of rib fractures, more research will estimate the incidence of missed injuries in less severe patients.

Limitations of this study is the small number of included cases without a control population. Patients with a missed diaphragm injury were not confirmed and a long-time follow-up and secondary surgical repair of those patients couldn't be compared with early on surgical repair. Another limitation was the need for a thoracic surgeon with sufficient experience in VATS to perform a thoracoscopy during SSFR, therefor only one patient underwent a minimal invasive technique while almost the complete cohort underwent a thoracotomy. So difference in approach for intrathoracic inspection could not be compared.

\section{Conclusions}

With the recent shift towards surgical stabilisation of rib fractures an inspection thoracoscopy or thoracotomy should be considered during SSFR in selected patients to minimize the incidence of missed injuries requiring early or late surgical treatment.

\section{Declarations}

Funding

Not applicable.

\section{Conflicts of Interest}

The authors have no relevant conflict of interest or financial disclosures.

\section{Ethical approval}

Not applicable due to the retrospective character of this study.

\section{Consent to participate}

Not applicable. 


\section{Consent for publication}

Not applicable.

\section{Availability of data and material}

Anonymous data set available when requested.

\section{Code availability}

Not applicable.

\section{Author Contribution}

Reinier de Groot, Wim Hogeboom and Pascal Steenvoorde designed the research. Tess Wemeijer and Dominique Withaar collected the data. All authors analysed the data and contributed to the writing of the manuscript.

\section{References}

1. Tanaka H, Yukioka T, Yamaguti Y, Shimizu S, Goto H, Mastuda H, Shimazaki S. Surgical stabilization of internal pneumatic stabilization? A prospective randomized study of management of severe flail chest patients. J Trauma. 2002 Apr;52(4):727-31. discussion 732.

2. Granetzny A, El-Aal MA, Emam ER, Shalaby A, Boseila A. Surgical versus conservative treatment of flail chest. Evaluation of pulmonary status. Interact Cardiovasc Thorac Surg. 2005;4:583-7.

3. Marasco SF, Davies AR, Cooper J, Varma D, Bennett V, Nevill R, Lee G, Bailey M, Fitzgerald M. Prospective Randomized Controlled Trial of Operative Rib Fixation in Traumatic Flail Chest. J Am Coll Surg. May 2013;216(5):924-32.

4. Kane ED, Jeremistky E, Pieracci FM, Majercik S, Doben AR. Quantifying and exploring the recent national increase in surgical stabilization of rib fractures. J Trauma Acute Care Surg. 2017;83(6):1047-52.

5. Pieracci FM, Leasia K, Bauman Z, Eriksson EA, Lottenberg L, Majercik S, Powell L, Sarani B, Semon G, Thomas B, et al. A multicentre, prospective, controlled clinical trial of surgical stabilization of rib fractures in patients with severe, nonflail fracture patterns. J Trauma Acute Care Surg. 2020, Volume 88, number 2:249-257.

6. Pfeifer R, Pape HC. Missed injuries in trauma patients: A literature review. Patient Saf Surg. 2008;2:20. 
7. Larici AR, Gotway MB, Litt HI, Reddy GP, Webb WR, Gotway CA, Dawn SK, Marder SR, Storto ML. Helical CT with sagittal and coronal reconstructions: accuracy for detection of diaphragmatic injury. AJR Am J Roentgenol. 2002;179:451-7.

8. Fair KA, Gordon NT, Barbosa RR, Rowell SE, Watters JM, Schreiber MA. Traumatic diaphragmatic injury in the American College of Surgeons National Trauma Data Bank: a new examination of a rare diagnosis. Am Journal Surg. 2015;209:864.

9. Baker SP, O'Neill B, Haddon W Jr, Long WB. The injury severity score: a method for describing patients with multiple injuries and evaluating emergency care. J Trauma. 1974 Mar;14(3):187-96.

10. Panda A, Kumar A, Gamanagatti S, Patil A, Kumar S, Gupta A. Traumatic diaphragmatic injury: a review of CT signs and the difference between blunt and penetrating injury. Diagn Interv Radiol. 2014 Mar-Apr;20(2):121-8.

11. Williams M, Bulger EM, Collins KA. Recognition and management of diaphragmatic injury in adults. UpToDate. Last updated: Mar 04, 2019. Accessed March 10th, 2020.

12. Pieracci FM, Majercik S, Ali-Osman F, Ang D, Doben A, Edwards JG, French B, Gasparri M, Marasco S, Minshall C, et al. Consensus Statement: Surgical Stabilization of Rib Fractures Rib Fracture Colloquium Clinical Practice Guidelines.Injury http://dx.doi.org/10.1016/j.injury.2016.11.026.

13. Schots JPM, Vissers YLJ, Hulsewé KWE, Meesters B, Hustinx PA, Pijnenburg A, Siebenga J, de Loos ER. Addition of Video-assisted Thorascopic Surgery to the Treatment of Flail Chest. Ann Thorac Surg. 2017;103:940-4.

14. Chou YP, Kuo LC, Soo KM, Tarng YW, Chiang HI, Huang FD, Lin HL. The role of repairing lunch laceration during video-assisted thorascopic surgery evacuations for retained haemothorax caused by blunt chest trauma. Eur J Cardiothorac Surgb2014;46:107 - 11. 